\title{
Decreased semaphorin 3A expression is associated with a poor prognosis in patients with epithelial ovarian carcinoma
}

\author{
HAIYAN JIANG ${ }^{1}$, LEI $^{2}{ }^{2}$, FEIRAN WANG $^{3}$, ZHICHAO SUN $^{4}$, ZHONGWEI HUANG $^{2}$ and QINGHUA XI ${ }^{1}$ \\ Departments of ${ }^{1}$ Obstetrics and Gynecology, ${ }^{2}$ Emergency Medicine and ${ }^{3}$ Surgery, Affiliated Hospital of Nantong University, \\ Nantong, Jiangsu 226001; ${ }^{4}$ Medical College, Nantong University, Nantong, Jiangsu 226019, P.R. China
}

Received July 4, 2014; Accepted March 9, 2015

DOI: $10.3892 / \mathrm{ijmm} .2015 .2142$

\begin{abstract}
Semaphorin 3A (SEMA3A) was initially identified to play an important role in axonal guidance. Recently, SEMA3A has also been considered as a candidate tumor suppressor, since it is often downregulated in numerous types of cancer, including prostate cancer, breast cancer and glioma. However, the biological role of SEMA3A in ovarian cancer is not clear. In the present study, the expression of SEMA3A in ovarian cancer and normal ovarian epithelial tissues was detected by immunofluorescence, reverse transcription-quantitative polymerase chain reaction (RT-qPCR) and western blotting, and the associations between the expression of SEMA3A with the development of ovarian cancer, clinicopathological characteristics and survival were also analyzed. Results from immunofluorescence, RT-qPCR and western blotting showed that SEMA3A is significantly downregulated in epithelial ovarian carcinoma compared to normal ovarian epithelial specimens $(\mathrm{P}<0.05)$. The expression levels of SEMA3A were lower in the cancer tissues with III/IV stage [the International Federation of Gynecology and Obstetrics (FIGO) stage], poor histological grade, lymph node metastasis and distant metastasis compared to that in the cancer tissues with I/II stage (FIGO), well histological grade, or without lymph node metastasis and distant metastasis $(\mathrm{P}<0.05)$. A decreased expression of SEMA3A is associated with a poor prognosis $(\mathrm{P}<0.001)$. The present findings suggest that decreased SEMA3A expression may be associated with the development of epithelial ovarian carcinoma, and therefore, SEMA3A may be a valuable prognostic marker, as well as a potential molecular therapy target for ovarian cancer patients.
\end{abstract}

Correspondence to: Professor Qinghua Xi, Department of Obstetrics and Gynecology, Affiliated Hospital of Nantong University, 20 West Temple Road, Nantong, Jiangsu 226001, P.R. China E-mail: lansyne@163.com

Professor Zhongwei Huang, Department of Emergency Medicine, Affiliated Hospital of Nantong University, 20 West Temple Road, Nantong, Jiangsu 226001, P.R. China

E-mail: tdfyhuangzw@163.com

Key words: semaphorin 3A, epithelial ovarian carcinoma, prognosis

\section{Introduction}

Epithelial ovarian carcinoma (EOC) is the fifth leading cause of cancer-related mortality and is one of the most aggressive tumors of all gynecological malignancies in Western countries. According to Cancer Statistics, it was estimated that approximately 15,500 individuals succumbed to the disease in the United States in 2012 (1). The majority of EOC patients have advanced intraperitoneal metastatic diseases at diagnosis, as this carcinoma frequently remains clinically silent. Since the treatment strategy consisting of maximum cytoreductive surgery followed by taxane plus platinum chemotherapy was established, the short-term prognosis of patients with EOC has improved. However, despite the high-level sensitivity of EOC to paclitaxel, the prognosis of advanced or recurrent cases remains poor, as the majority of mortality cases are the result of metastasis, which is refractory to these chemotherapeutic agents. Although various additional molecular-targeting therapies, such as the use of anti-angiogenic agents, have been investigated in order to overcome paclitaxel resistance, the effect of this treatment is limited $(2,3)$. Currently, numerous studies have investigated new methods and targets to treat this disease (4-6).

Recently, increasing attention has been paid to the association between the nervous system and cancer, as increasing evidence supports that common genetic mechanisms are involved in cancer development and the progression of neurodegenerative disease (7). The nervous system may exert a potential influence on cancer development; environmental enrichment has been shown to significantly inhibit xenograft tumor growth, but the mechanism remains elusive (8). Members of the semaphorin (SEMA) family, which were originally reported as axon guidance molecules $(9,10)$, have gained increasing attention recently due to their roles in tumor growth and metastasis (1-13). SEMAs can be classified into eight classes (SEMA1-7 and viral SEMA). Class 3 SEMAs (SEMA3) are the only secreted SEMAs in vertebrates. Several class 3 SEMAs, including SEMA3A, SEMA3B, SEMA3E and SEMA3F, have been characterized as anti-angiogenic agents (14-19). For example, SEMA3B, SEMA3F and SEMA4D regulate tumor angiogenesis, growth and metastasis in different manners $(20,21)$. Previous studies showed that SEMA3A, which is considered as the candidate tumor suppressor, is often downregulated in numerous types of cancer, including 
prostate cancer, breast cancer and glioma (22-24). However, whether SEMA3A is also downregulated in epithelial ovarian carcinoma remains unclear. Therefore, the present study focused on the expression of SEMA3A in epithelial ovarian carcinoma and the potential contribution of SEMA3A in the prediction of prognosis.

\section{Materials and methods}

Patient information and tissue sampling. A total of 125 specimens of epithelial ovarian carcinoma from patients diagnosed between 2000 and 2010 were obtained from surgery in the Department of Obstetrics and Gynecology and the Department of Pathology in the Affiliated Hospital of Nantong University (Nantong, China). None of the patients had received any form of tumor-specific therapy prior to surgery. Samples were collected (median age, 59 years; range, 33-82 years), and according to the classification of the International Federation of Gynecology and Obstetrics (FIGO) in 2009, there were 20 cases of stage I, 39 stage II, 35 stage III and 37 stage IV. The histological grade of the tumor was classified as GI (well-differentiated) in 55 cases, GII (moderately differentiated) in 32 cases and GIII (poorly differentiated) in 38 cases. Of all the samples, there were 72 cases with lymphatic metastasis (median age, 55 years; range, 39-75 years), 58 with pelvic metastasis (median age, 53 years; range, $42-79$ years) and 53 with peritoneal metastasis (median age, 56; range, 35-68 years). The follow-up period ranged from 2 to 60 months with an average of 29.7 months and a median of 20 months. The 15 cases of normal ovarian epithelium specimens were obtained from preventive excision of the uterus and accessories. All the tissues were obtained with the consent of the patients. The study protocol followed the guidelines in the Helsinki Declaration and was approved by the ethics committee (Institutional Review Board) of Nantong University.

Double-labeling immunofluorescence staining and confocal microscopy. All the specimens were embedded in optimum cutting temperature compound and frozen in liquid nitrogen cooled 2-methylbutane. The samples were subsequently divided into $20-\mu \mathrm{m}$ sections using a cryostat. Sections were fixed with cold acetone, blocked with $10 \%$ bovine serum albumin (BSA) in phosphate-buffered saline (PBS) containing $0.2 \%$ triton $\mathrm{X}-100$, and further permeabilized/blocked in the blocking solution (5\% BSA in PBS containing $0.3 \%$ Triton X-100) for $1 \mathrm{~h}$ at room temperature. Sections were first blocked with $10 \%$ BSA to prevent non-specific binding and incubated with a goat polyclonal SEMA3A primary antibody (SC-1148, 1:100; Santa Cruz Biotechnology, Inc., Dallas, TX, USA) overnight at $4^{\circ} \mathrm{C}$, followed by rabbit anti-goat $\operatorname{IgG}$ (H\&L) secondary antibody (FITC; 1:100; Abnova Corporation, Taipei, Taiwan) for $2 \mathrm{~h}$ at room temperature in a humidified chamber with minimal exposure to light. 4'6-Diamidino-2-phenylindole (DAPI) (Sigma-Aldrich, St. Louis, MO, USA) was used to visualize nuclei and all the washes were performed in $1 \mathrm{X}$ PBS. The sample images were captured using a confocal microscope. Sections were analyzed with a Leica SP5 high-speed spectral confocal laser-scanning microscope (Leica Microsystems, Wetzlar, Germany) or a Zeiss LSM 710 confocal microscope (Carl Zeiss, Oberkochen, Germany).
Immunofluorescence staining for single- or double-contractile markers was performed using randomly selected slides (4-5 slides per each eye) containing four sections per slide and was examined under the confocal microscope. Specific fluorescence was captured by confocal microscopy with exposure time kept constant across all the images. Immunoreactivity was evaluated by the quantification and stereological counting procedure, as well as semi-quantitative evaluation using the immunofluorescence staining intensity score and distribution score.

From the quantification and stereological counting procedure, 16-bit image sections were analyzed by NIH Image $\mathbf{J}$ software (National Institutes of Health). Fluorescence intensity of pixel gray values in eight separate regions of interest per region of the normal and tumor tissues was calculated and averaged across each tissue region. This was performed separately for each label (SEMA3A and DAPI). The fluorescence intensity for SEMA3A in normal and tumor tissues was subsequently compared using analysis of variance and Tukey's and Sidak's comparison tests.

For the semi-quantitative evaluation method, the immunoreactive score was defined as the proportion score multiplied by the intensity score, according to the way of evaluation of immunohistochemistry (IHC). The proportion scores were defined as 0 , negative; $1,<10 \% ; 2,11-50 \% ; 3,51-80 \%$; and $4,>80 \%$ positive cells. The intensity scores were defined as 0 , negative; 1 , weak; 2 , moderate; and 3 , strong. The total score ranged from 0 to 12 . The immunoreactivity scores were divided into one of the following three groups based on the final score; negative immunoreactivity was defined as a total score of 0 , moderate expression was defined as a total score of $1-4$, and strong expression was defined as a total score of $>4$.

Western blot analysis. Total protein was extracted by a lysis buffer containing protease inhibitors (Promega, Madison, WI, USA). Equal amounts of protein were separated by $10 \%$ sulfate polyacrylamide gel electrophoresis and subsequently transferred to a polyvinylidene fluoride membrane. The membrane was blocked for $2 \mathrm{~h}$ with $5 \%$ skimmed milk in TBS (Tris-buffered saline). After incubation with the primary antibodies overnight at $4^{\circ} \mathrm{C}$ [goat polyclonal SEMA3A primary antibody (SC-1148, 1:200) or a goat anti- $\beta$-actin as internal reference (1:2000; Sigma-Aldrich)], membranes were washed for 5 min with TBS containing $0.1 \%$ Tween-20 three times and subsequently incubated with horseradish peroxidase-coupled mouse anti-rabbit/goat IgG antibodies (1:1,000; AB Biotec, Stockholm, Sweden) for $2 \mathrm{~h}$ at room temperature. Signals were detected using electrochemiluminescence (Pierce Corp., Rockford, IL, USA) followed by film development.

Expression analysis by reverse transcription-quantitative polymerase chain reaction $(R T-q P C R)$. The mRNA expression of SEMA3A was analyzed by RT-qPCR. Total RNA was extracted using the TRIzol reagent (Gibco Life Technologies, Beijing, China). RT-qPCR was performed using the HotStart-IT SYBR Green qPCR Master mix (2X; USB Corp., Cleveland, $\mathrm{OH}, \mathrm{USA})$. According to the manufacturer's instructions, $25 \mu \mathrm{l}$ reactions were carried out with $25 \mu \mathrm{l}$ of cDNA. RT-qPCR experiments were performed in a LightCycler 480 system (Roche Applied Sciences, Basal, Switzerland). Cycling param- 

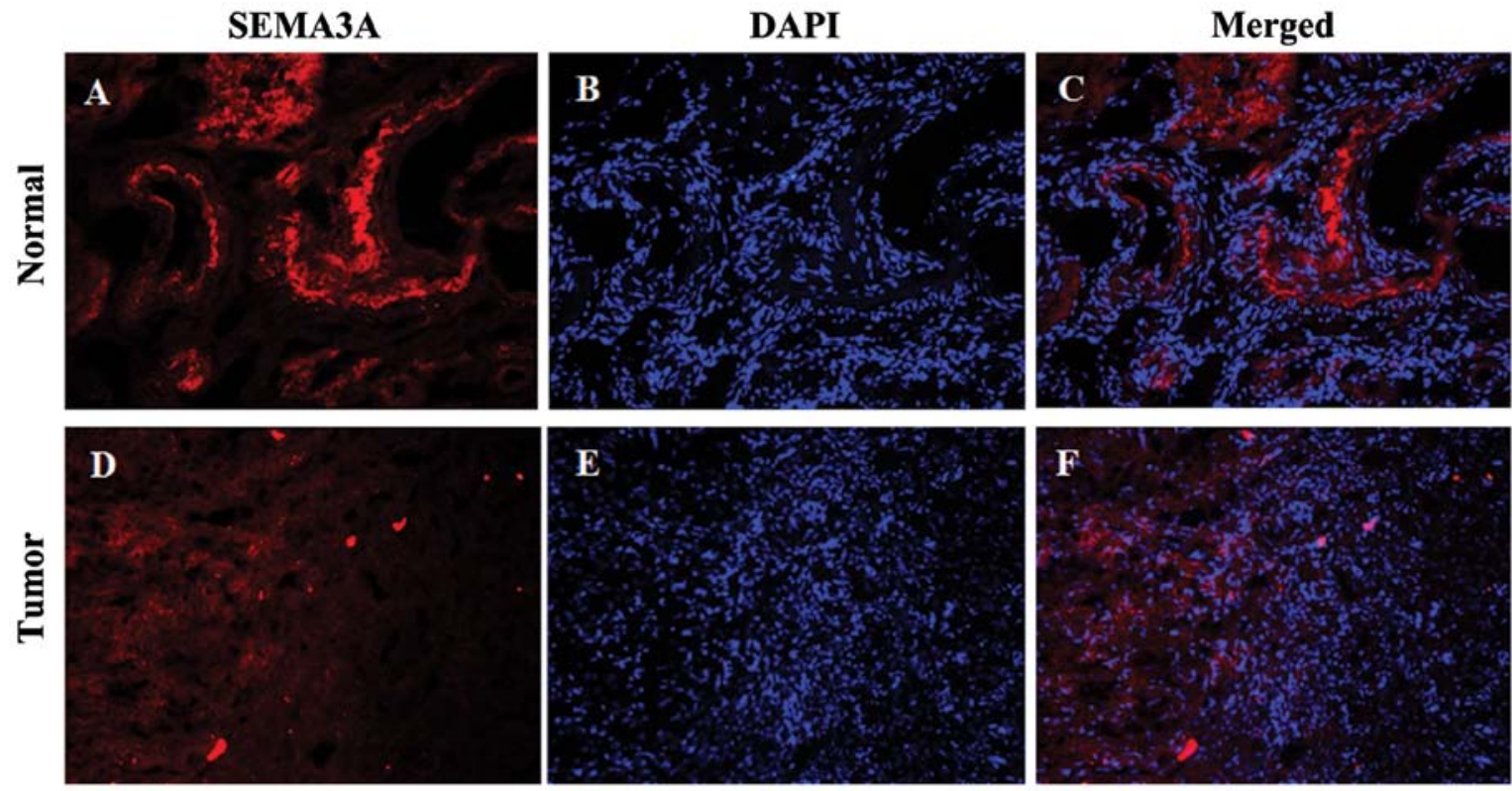

Figure 1. SEMA3A expression in normal ovarian epithelium and epithelial ovarian carcinoma. (A) SEMA3A expression in normal tissues; (B) DAPI in normal tissues; (C) merged image of A and B; (D) SEMA3A expression in tumor tissues; (E) DAPI in tumor tissues; and (F) merged image of D and E showing differentially expressed proteins in normal ovarian epithelium and epithelial ovarian carcinoma, respectively. The color intensity indicates the degree of protein regulation. SEMA3A, semaphorin 3A; DAPI, 4'6-diamidino-2-phenylindole.

eters were as follows: Hot start at $95^{\circ} \mathrm{C}$ for $10 \mathrm{~min} ; 40$ cycles of amplification/quantification at $95^{\circ} \mathrm{C}$ for $10 \mathrm{sec}, 60^{\circ} \mathrm{C}$ for $30 \mathrm{sec}$ and $72^{\circ} \mathrm{C}$ for $30 \mathrm{sec}$, during which time fluorescence was measured. Melting curve analysis was performed using continuous fluorescence acquisition from $65-97^{\circ} \mathrm{C}$. These cycling parameters generated single amp icons for the two primer sets used according to the presence of a single melt peak. GAPDH was selected as the internal reference. All RT-qPCR reactions were repeated three times for each gene and each sample was performed in triplicate. Sequences of the primers for SEMA3A were: Forward, 5'-ATCTGTATCAGGTGCCTCTTACC-3'; and reverse, 5'-TCTCAACGAATCGTCTTAGGAC-3'. The relative changes in gene expression were analyzed by the $2^{-\Delta \Delta C T}$ method. Triplicates were performed for each sample in three independent experiments.

Clinicopathological analysis. The mRNA expression levels of SEMA3A in ovarian tissues were used to analyze the association between SEMA3A expression and clinicopathological characteristics, as well as the survival time of the patients. Pathological analysis was performed by the Departments of Pathology of Nantong University, and validated by qualified experts. During the follow-up period, overall survival was measured from diagnosis to fatality or to the last follow-up (at five years). At the time of analysis, 86 patients (68.8\%) succumbed, 37 patients (29.6\%) were alive, and 2 patients were lost during the follow-up. The estimated median survival time for all patients was 28 months, and the calculated survival rates were $72.8 \%$ at 1 year, $48.0 \%$ at 2 years, and $29.6 \%$ at 5 years.

Post-operative follow-up. Following surgery, each patient was scheduled for a follow-up examination, including physical examination, complete blood count, tumor markers' tests and ultrasound scan of the pelvis every 3 months in the first year, semi-annually in the second year, and annually after 3 years.
More frequent examinations were scheduled when clinically indicated. The cause of mortality was registered and classified as mortality due to this cancer, other causes or unknown causes. Fatality of a patient was ascertained by reporting from the family and verified by a review of public records.

Statistical analysis. Tukey's and Sidak's comparison tests were used to compare the fluorescence intensity. SPSS 19.0 statistical software (IBM Corp., Armonk, NY, USA) was adopted for data analysis. Counting data comparisons between groups were subjected to the $\chi^{2}$ test. Survival analysis was computed by means of the Kaplan-Meier method and significant levels were assessed using the log-rank test. The results are expressed as the means \pm standard deviation of at least three independent experiments, and for all statistical analyses, $\mathrm{P}<0.05$ was considered to indicate a statistically significant difference.

\section{Results}

SEMA3A is detected at a lower level in epithelial ovarian carcinoma. SEMA3A was detected primarily in the nucleus and cytoplasm of the normal ovarian epithelium (Fig. 1A and C). Only 2 (13.3\%) of the 15 normal ovarian epithelium showed moderate intensity, while $13(86.7 \%)$ showed a strong intensity (Table I). However, among the 125 epithelial ovarian carcinoma cases, moderate intensity was observed in 78 (62.4\%), and a strong intensity was observed in 47 (37.6\%) (Table I). Quantitative fluorescence intensity of SEMA3A was lower in the tumor compared to the normal specimens (Fig. 2). There was a significant difference in the expression of SEMA3A between normal and tumor tissues $(\mathrm{P}<0.001$; Table I and Fig. 2).

SEMA3A protein expression is downregulated in ovarian carcinoma tissues. The protein expression of SEMA3A was 
Table I. Expression of SEMA3A in normal ovarian epithelum and epithelial ovarian carcinoma.

\begin{tabular}{lrrrr}
\hline & & \multicolumn{3}{c}{ SEMA3A } \\
\cline { 3 - 5 } Tissue & No. & M & S & P-value \\
\hline Tumor & 125 & 78 & 47 & $<0.001$ \\
Normal & 15 & 2 & 13 & \\
\hline
\end{tabular}

SEMA3A, semaphorin 3A; No., number of patients; M, moderate; S, strong.

Table II. mRNA expression of SEMA3A in normal ovarian epithelum and epithelial ovarian carcinoma.

\begin{tabular}{lccc}
\hline Gene & $\begin{array}{c}\text { Normal, median } \\
\text { copies } / \mu 1 \text { (range) }\end{array}$ & $\begin{array}{c}\text { Tumor, median } \\
\text { copies } / \mu 1 \text { (range) }\end{array}$ & P-value \\
\hline SEMA3A & $171(49-349)$ & $51(3-112)$ & $<0.001$ \\
\hline
\end{tabular}

SEMA3A, semaphorin 3A.

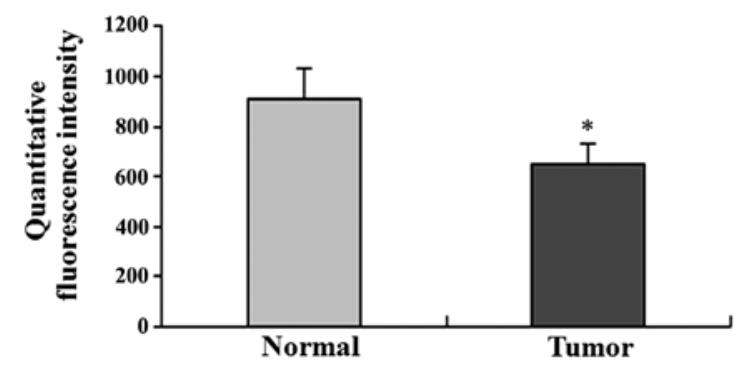

Figure 2. Quantitative fluorescence intensity of normal ovarian epithelium and epithelial ovarian carcinoma.

examined by western blot analysis in ovarian tumors and normal ovarian epithelium, as performed on all the epithelial ovarian carcinoma and the normal ovarian epithelial tissues. The relative quantity of SEMA3A protein expression was normalized to $\beta$-actin. Five pairs of cancerous and normal ovarian tissues were randomly selected and presented in Fig. 3A, while summary data are presented in Fig. 3B. The expression of the SEMA3A protein was downregulated in the majority of the samples of ovarian tumors compared to in the normal tissues (Fig. 3A). In extremely few cases, such as the second pair (2\#) in Fig. 3A, it appeared that the expression of SEMA3A in the tumor was close to the normal tissue (Fig. 3A). The average SEMA3A protein level in the epithelial ovarian carcinoma was significantly lower than that in the normal ovarian epithelial tissues $(\mathrm{P}<0.05$; Fig. 3B).

SEMA3A mRNA expression is downregulated in ovarian carcinoma tissues. In addition to the protein expression of SEMA3A, the mRNA expression was also detected in the epithelial ovarian carcinoma and normal ovarian epithelial tissues. As shown in Table II, the mRNA expression of SEMA3A (median, 51 copies $/ \mu 1$; range from 5 to 112 copies $/ \mu$ l) was
A

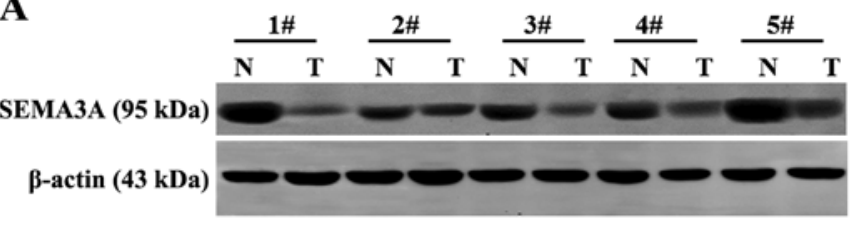

B

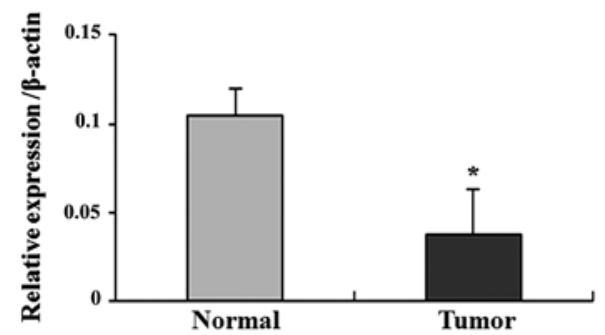

Figure 3. Protein expression of SEMA3A in normal ovarian epithelium and epithelial ovarian carcinoma. (A) Representative results of SEMA3A protein expression. (B) Relative SEMA3A protein expression levels was markedly decreased in the tumor tissues compared to in the normal specimens. SEMA3A, semaphorin 3A; N, normal; T, tumor.

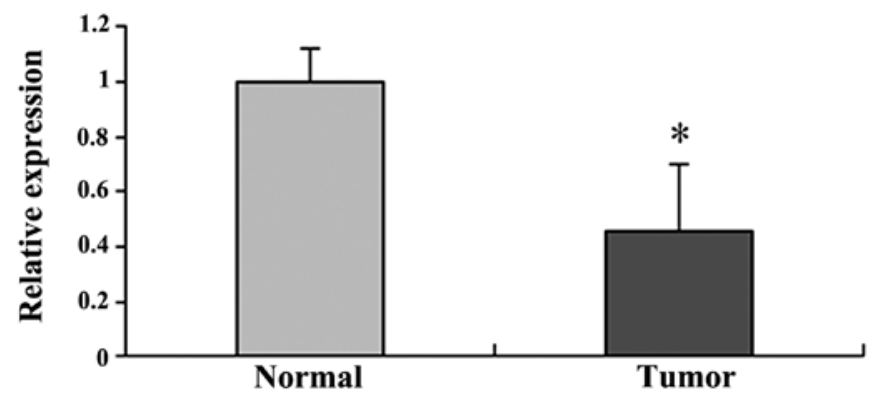

Figure 4. Quantitative reverse transcription PCR analysis of SEMA3A expression in normal ovarian epithelium and epithelial ovarian carcinoma. The relative mRNA expression of SEMA3A was significantly lower in the tumor tissues compared to the normal specimens. PCR, polymerase chain reaction; SEMA3A, semaphorin 3A.

significantly reduced in ovarian carcinoma samples compared to the normal samples (median, 171 copies $/ \mu 1$; range from 49 to 349 copies/ $\mu 1)(\mathrm{P}<0.001)$. Quantification of SEMA3A mRNA expression revealed a significant decrease in cancerous compared to normal tissues (Table II and Fig. 4).

The association between SEMA3A mRNA expression levels of the ovarian tumors and clinicopathological characteristics are presented in Table III.

Correlations between the RT-qPCR results of SEMA3A expression in ovarian tumor tissues and various clinicopathological characteristics of patients were analyzed by $\chi^{2}$ test and are listed in Table III. Using the quartile limits of mRNA expression to divide patient population into low and high producers allowed the interquartile range to be set as a cut-off and a significant correlation between the mRNA expression of SEMA3A and clinicopathological characteristics to be established. Median expression of SEMA3A in cancerous tissues was 51 copies $/ \mu 1$, dividing the samples into two groups: The negative ( $\leq 51 \mathrm{copies} / \mu \mathrm{l}$ ) and positive expression groups of SEMA3A ( $>51$ copies $/ \mu 1)$.

The downregulation of SEMA3A significantly correlated with FIGO stage, histological grade, lymphatic metastasis and 
Table III. Association between SEMA3A expression levels of epithelial ovarian carcinoma and clinicopathological factors.

\begin{tabular}{lrrrr}
\hline & & \multicolumn{3}{c}{ SEMA3A } \\
\cline { 4 - 5 } Characteristics & No. & N & P & P-value \\
\hline Age, years & & & & \\
$\leq 59$ & 63 & 36 & 27 & 0.070 \\
$>59$ & 62 & 42 & 20 & \\
Tumor size, cm & & & & \\
$\leq 2$ & 45 & 25 & 20 & 0.076 \\
$>2$ & 80 & 53 & 27 & \\
FIGO stage & & & & \\
I/II & 57 & 31 & 26 & 0.035 \\
III/IV & 68 & 47 & 21 & \\
Histogical grade & & & & \\
$\quad$ Well & 55 & 25 & 30 & 0.000 \\
$\quad$ Moderate + poor & 70 & 53 & 17 & \\
Histotype & & & & \\
$\quad$ Serous & 58 & 35 & 23 & 0.496 \\
$\quad$ Mucinous & 48 & 33 & 15 & \\
Endometroid & 14 & 7 & 7 & \\
Clear cell & 3 & 2 & 1 & \\
$\quad$ Undifferentiated & 2 & 1 & 1 & \\
Lymph node metastasis & & & & \\
$\quad$ Negative & 53 & 26 & 27 & 0.004 \\
Positive & 72 & 52 & 20 & \\
Distant metastasis & & & & \\
$\quad$ Negative & 67 & 47 & 20 & 0.024 \\
Positive & 58 & 31 & 27 & \\
\hline
\end{tabular}

SEMA3A, semaphorin 3A; No., number of patients; N, negative; $\mathrm{P}$, positive; FIGO, International Federation of Gynecology and Obstetrics.

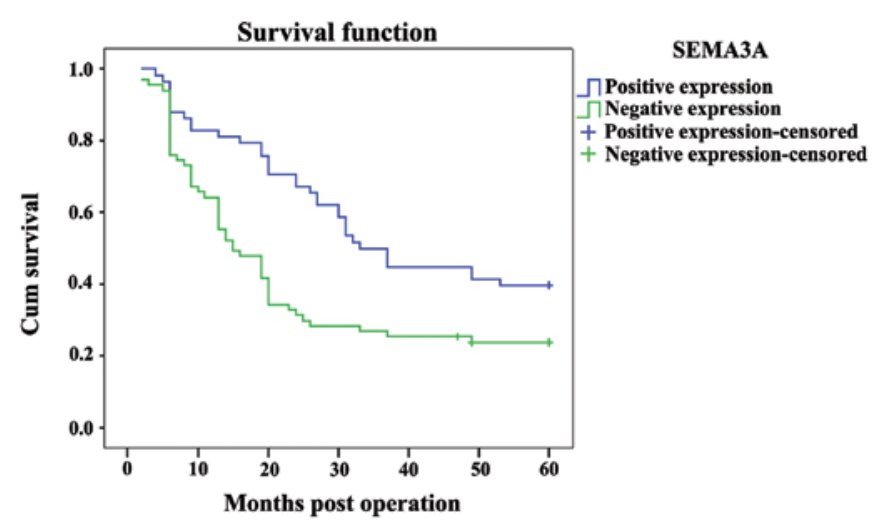

Figure 5. Kaplan-Meier survival curves of cancer patients following surgical resection. The cancer patients were divided into negative and positive expression groups of SEMA3A. Negative expression of SEMA3A was strongly associated with worse patient survival. SEMA3A, semaphorin 3A.

distant metastasis $(\mathrm{P}<0.05)$. However, there was no significant correlation between SEMA3A expression and age, tumor size or histological type ( $>0.05$; Table III).
Expression of SEMA3A is associated with the survival rate of ovarian carcinoma patient. The prognostic role of SEMA3A on the overall survival rate of ovarian carcinoma patients was investigated by comparing the 5-year survival rate of patients with high or low levels of SEMA3A expression in tumors using Kaplan-Meier survival curves and the log-rank test. There were 58 cases in the positive SEMA3A expression group ( $>51$ copies $/ \mu 1$ ), of which 35 succumbed, and the 5 -year overall survival rate was $39.7 \%$. In the negative SEMA3A expression group ( $<51$ copies $/ \mu$ l) there were 67 cases, of which 51 succumbed and 2 were lost during follow-up. The 5-year overall survival rate for the negative group was $20.9 \%$. The overall survival rate of the high SEMA3A expression group was significantly longer than that of the low SEMA3A expression group $(\mathrm{P}<0.001 ;$ Fig. 5$)$.

\section{Discussion}

SEMA is a multifunctional protein whose function includes, but is not limited to, axonal guidance $(25,26)$. Class 3 SEMAs, such as SEMA3A, SEMA3B and SEMA3F, have been previously characterized as natural tumor suppressors and there are indications that SEMA3E may also function as a natural tumor suppressor (17,27-30). Previous studies have also shown that SEMAs function as potent inhibitors of angiogenesis $(15,19)$. The expression of these SEMAs in several types of breast cancer-derived tumor cells can inhibit the growth of tumors following the subcutaneous implantation of these cells (13). SEMA3A is considered to be a candidate tumor suppressor in certain types of cancer, as it inhibits the proliferation of malignant mesothelium cells, decreases the adhesion or migration of prostate or breast cancer cells, and promotes apoptosis in leukemic T cells (7). However, the roles of SEMA3A in patients with ovarian cancer have not been extensively studied. Ovarian cancer, having the highest fatality rate of the female reproductive diseases, is the leading type with the prominent features of hidden onset, malignancy, easy metastasis of the normal tissue adjacent to ovarian cancer tissue and lack of effective early screening methods (1).

The present study focused on the expression of SEMA3A in the most common type of ovarian cancer, epithelial ovarian carcinoma. From the immunofluorescence staining results, there was a significant difference in the expression of SEMA3A between normal and cancerous tissues. The protein and mRNA expression of SEMA3A in ovarian carcinoma tissues and in normal tissues was also determined. As expected, the average SEMA3A protein level in the epithelial ovarian carcinoma was significantly lower than that in the normal ovarian epithelial tissue, while the mRNA expression of SEMA3A was only $27 \%$ in cancerous compared to normal tissues. The correlations between SEMA3A mRNA expression in ovarian tumor tissues and various clinicopathological characteristics of patients were analyzed by $\chi^{2}$ test. The downregulation of SEMA3A significantly correlated with FIGO stage, histological grade, lymphatic metastasis and distant metastasis. However, there was no significant correlation between SEMA3A expression and age or tumor size. The overall survival rate of the positive SEMA3A expression group was significantly longer than that of the negative SEMA3A expression group. The discrepant changes for SEMA3A in ovarian cancer in the present study 
indicate the important role of SEMA3A in the development of epithelial ovarian carcinoma. Additionally, decreased expression of SEMA3A was correlated with poor prognosis in ovarian tumor, suggesting that SEMA3A may be an inhibitor in ovarian epithelial cancer. SEMA3A has been indicated as a tumor suppressor in other types of cancer (22,30-33). The mechanism of the inhibitory role of SEMA3A may be associated with its interaction with integrins. For example, in breast cancer, SEMA3A inhibits cell attachment and cell migration by affecting the activation or the stabilization of surface integrins. Inhibition of integrins by SEMA3A resulted in a blockade of endothelial and tumor cell migration, leading to reduced tumor angiogenesis and metastasis $(34,35)$. The present results may reflect the ability of SEMA3A to block tumor cell migration and metastasis. In addition, a more recent study on microRNA (miR) observed that the upregulation of miR-30b/30d correlates with a higher metastatic potential, shorter time to recurrence and reduced overall survival time. Among the target genes of miR-30b/30d, the investigators identified a significant downregulation of SEMA3A (36). Taken together with the present results, these data indicate that SEMA3A may be involved in cancer metastasis. The ability of SEMA3A to inhibit tumor angiogenesis by competing with vascular endothelium growth factor for binding with neuropilin 1 has been more intensively studied in colorectal cancer. Therefore, much remains to be studied regarding the exact role of SEMA3A in different types of cancer.

Although significant results of SEMA3A in epithelial ovarian carcinoma were obtained, there were only 15 cases of normal tissues. More cases are required in future studies as it is possible that this affected the results.

Double-labeling immunofluorescence staining and confocal microscopy were used instead of IHC, which is a common technique used for diagnostic and research purposes. IHC is one of the most important methods in pathology due to its central role in the classification of diseases by the evaluation of receptors and other cellular components in biopsies and surgical resections. IHC involves staining a thin representative tissue section to evaluate the intensity and localization of the staining in order to understand antigen expression. Estimation of the distribution and the expression is subjectively performed by trained investigators through visual inspection using a microscope, and staining is commonly reported as,,-+++ and +++ . The technique provides superior spatial resolution, but is operator-dependent and further relies on multi-layered end-point measurements that increase inaccuracy (37). By contrast, fluorescence objectively reflected the expression under the conditions with the case.

In the present study, the IHC method and the measurement of quantitative fluorescence intensity was applied, providing a new method for the evaluation of immunoreactivity, particularly when there were multiple targets and antibody labeling. Quantitative mRNA expression was also used to analyze the association of SEMA3A with clinicopathological characteristics, as well as the overall survival rate. Using the quartile limits of mRNA expression to divide the population of patients into low and high groups allowed a significant correlation between mRNA expression and clinicopathological characteristics to be established, as well as survival rate, which more accurately reflected the real situation.
In conclusion, SEMA3A was downregulated in human epithelial ovarian carcinoma, and the decreased SEMA3A expression was strongly associated with worse patient survival. Therefore, SEMA3A could be used as a valuable prognostic marker, as well as a potential molecular therapy target for ovarian cancer.

\section{Acknowledgements}

The present study was supported by the Natural Science Foundation of Jiangsu Province (grant no. BK20131199).

\section{References}

1. Siegel R, Naishadham D and Jemal A: Cancer statistics, 2012. CA Cancer J Clin 62: 10-29, 2012.

2. Teoh D and Secord AA: Antiangiogenic agents in combination with chemotherapy for the treatment of epithelial ovarian cancer. Int J Gynecol Cancer 22: 348-359, 2012.

3. Usha L, Sill MW, Darcy KM, et al: A Gynecologic Oncology Group phase II trial of the protein kinase C-beta inhibitor, enzastaurin and evaluation of markers with potential predictive and prognostic value in persistent or recurrent epithelial ovarian and primary peritoneal malignancies. Gynecol Oncol 121: 455-461, 2011.

4. Wu H, Yao L, Mei J and Li F: Development of synthetic of peptide-functionalized liposome for enhanced targeted ovarian carcinoma therapy. Int J Clin Exp Pathol 8: 207-216, 2015.

5. Kannan K, Coarfa C, Chao PW, et al: Recurrent BCAM-AKT2 fusion gene leads to a constitutively activated AKT2 fusion kinase in high-grade serous ovarian carcinoma. Proc Natl Acad Sci USA: Mar 2, 2015 (Epub ahead of print).

6. Gurler H, Yu Y, Choi J, Kajdacsy-Balla AA and Barbolina MV: Three-dimensional collagen type I matrix up-regulates nuclear isoforms of the microtubule associated protein tau implicated in resistance to Paclitaxel therapy in ovarian carcinoma. Int J Mol Sci 16: 3419-3433, 2015.

7. Morris LG, Veeriah S and Chan TA: Genetic determinants at the interface of cancer and neurodegenerative disease. Oncogene 29: 3453-3464, 2010

8. Cao L, Liu X, Lin EJ, et al: Environmental and genetic activation of a brain-adipocyte BDNF/leptin axis causes cancer remission and inhibition. Cell 142: 52-64, 2010.

9. Chilton JK: Molecular mechanisms of axon guidance. Dev Biol 292: 13-24, 2006.

10. Dickson BJ: Molecular mechanisms of axon guidance. Science 298: 1959-1964, 2002.

11. Bielenberg DR, Hida Y, Shimizu A, et al: Semaphorin 3F, a chemorepulsant for endothelial cells, induces a poorly vascularized, encapsulated, nonmetastatic tumor phenotype. J Clin Invest 114: 1260-1271, 2004.

12. Christensen C, Ambartsumian N, Gilestro G, et al: Proteolytic processing converts the repelling signal Sema3E into an inducer of invasive growth and lung metastasis. Cancer Res 65: 6167-6177, 2005.

13. Neufeld G and Kessler O: The semaphorins: versatile regulators of tumour progression and tumour angiogenesis. Nat Rev Cancer 8: 632-645, 2008.

14. Kigel B, Varshavsky A, Kessler O and Neufeld G: Successful inhibition of tumor development by specific class-3 semaphorins is associated with expression of appropriate semaphorin receptors by tumor cells. PLoS One 3: e3287, 2008.

15. Kessler O, Shraga-Heled N, Lange T, et al: Semaphorin-3F is an inhibitor of tumor angiogenesis. Cancer Res 64: 1008-1015, 2004.

16. Casazza A, Fu X, Johansson I, et al: Systemic and targeted delivery of semaphorin $3 \mathrm{~A}$ inhibits tumor angiogenesis and progression in mouse tumor models. Arterioscler Thromb Vasc Biol 31: 741-749, 2011.

17. Maione F, Molla F, Meda C, et al: Semaphorin 3A is an endogenous angiogenesis inhibitor that blocks tumor growth and normalizes tumor vasculature in transgenic mouse models. J Clin Invest 119: 3356-3372, 2009.

18. Sakurai A, Gavard J, Annas-Linhares Y, et al: Semaphorin 3E initiates antiangiogenic signaling through plexin D1 by regulating Arf6 and R-Ras. Mol Cell Biol 30: 3086-3098, 2010. 
19. Varshavsky A, Kessler O, Abramovitch S, et al: Semaphorin-3B is an angiogenesis inhibitor that is inactivated by furin-like pro-protein convertases. Cancer Res 68: 6922-6931, 2008.

20. Rolny C, Capparuccia L, Casazza A, et al: The tumor suppressor semaphorin $3 \mathrm{~B}$ triggers a prometastatic program mediated by interleukin 8 and the tumor microenvironment. J Exp Med 205: 1155-1171, 2008.

21. Zheng C, Zhou Q, Wu F, et al: Semaphorin3F down-regulates the expression of integrin alpha(v)beta3 and sensitizes multicellular tumor spheroids to chemotherapy via the neuropilin-2 receptor in vitro. Chemotherapy 55: 344-352, 2009.

22. Bagci T, Wu JK, Pfannl R, Ilag LL and Jay DG: Autocrine semaphorin 3A signaling promotes glioblastoma dispersal. Oncogene 28: 3537-3550, 2009.

23. Li K, Chen MK, Li LY, et al: The predictive value of semaphorins 3 expression in biopsies for biochemical recurrence of patients with low- and intermediate-risk prostate cancer. Neoplasma 60: 683-689, 2013.

24. Staton CA, Shaw LA, Valluru M, et al: Expression of class 3 semaphorins and their receptors in human breast neoplasia. Histopathology 59: 274-282, 2011.

25. Neufeld G, Shraga-Heled N, Lange T, Guttmann-Raviv N, Herzog Y and Kessler O: Semaphorins in cancer. Front Biosci 10: 751-760, 2005.

26. Goshima Y, Ito T, Sasaki Y and Nakamura F: Semaphorins as signals for cell repulsion and invasion. J Clin Invest 109: 993-998, 2002

27. Tomizawa Y, Sekido Y, Kondo M, et al: Inhibition of lung cancer cell growth and induction of apoptosis after reexpression of 3 p21.3 candidate tumor suppressor gene SEMA3B. Proc Nat Acad Sci USA 98: 13954-13959, 2001.

28. Xiang R, Davalos AR, Hensel CH, Zhou XJ, Tse C and Naylor SL: Semaphorin 3F gene from human 3p21.3 suppresses tumor formation in nude mice. Cancer Res 62: 2637-2643, 2002.
29. Vacca A, Scavelli C, Serini G, et al: Loss of inhibitory semaphorin 3A (SEMA3A) autocrine loops in bone marrow endothelial cells of patients with multiple myeloma. Blood 108: 1661-1667, 2006

30. Moriya J, Minamino T, Tateno K, et al: Inhibition of semaphorin as a novel strategy for therapeutic angiogenesis. Circ Res 106: 391-398, 2010.

31. Pan H, Wanami LS, Dissanayake TR and Bachelder RE: Autocrine semaphorin3A stimulates alpha2 beta1 integrin expression/function in breast tumor cells. Breast Cancer Res Treat 118: 197-205, 2009

32. Herman JG and Meadows GG: Increased class 3 semaphorin expression modulates the invasive and adhesive properties of prostate cancer cells. Int J Oncol 30: 1231-1238, 2007.

33. Catalano A, Caprari P, Rodilossi S, et al: Cross-talk between vascular endothelial growth factor and semaphorin-3A pathway in the regulation of normal and malignant mesothelial cell proliferation. FASEB J 18: 358-360, 2004.

34. Oinuma I, Ishikawa $\mathrm{Y}$, Katoh $\mathrm{H}$ and Negishi M: The Semaphorin 4D receptor Plexin-B1 is a GTPase activating protein for R-Ras. Science 305: 862-865, 2004.

35. Toyofuku T, Yoshida J, Sugimoto T, et al: FARP2 triggers signals for Sema3A-mediated axonal repulsion. Nat Neurosci 8: $1712-1719,2005$.

36. Gaziel-Sovran A, Segura MF, Di Micco R, et al: miR-30b/30d regulation of GalNAc transferases enhances invasion and immunosuppression during metastasis. Cancer Cell 20: 104-118, 2011.

37. Dubois L, Andersson K, Asplund A and Bjorkelund H: Evaluating real-time immunohistochemistry on multiple tissue samples, multiple targets and multiple antibody labeling methods. BMC Res Notes 6: 542, 2013. 